Research paper

\title{
Bifurcation scenario of Turing patterns in prey-predator model with nonlocal consumption in the prey dynamics
}

\author{
N. Mukherjee ${ }^{\mathrm{a}, *}$, V. Volpert $^{\mathrm{b}, \mathrm{c}, \mathrm{d}}$ \\ a Institute of Mathematical Sciences, Chennai, India \\ ${ }^{\mathrm{b}}$ Institut Camille Jordan, UMR 5208 CNRS, University Lyon 1, Villeurbanne 69622, France \\ ${ }^{\mathrm{c}}$ INRIA, Université de Lyon, Université Lyon 1, Institut Camille Jordan 43 Bd. du 11 Novembre 1918, Villeurbanne Cedex 69200, France \\ d Peoples Friendship University of Russia (RUDN University) 6 Miklukho-Maklaya St, Moscow, 117198, Russian Federation
}

\section{A R T I C L E I N F O}

\section{Article history:}

Received 13 August 2020

Revised 13 December 2020

Accepted 18 December 2020

Available online 21 December 2020

\section{Keywords:}

Nonlocal model

Turing patterns

Prey-predator

Bifurcation

\begin{abstract}
A B S T R A C T
A prey-predator model with a sexual reproduction in prey population and nonlocal consumption of resources by prey in two spatial dimensions is considered. Patterns produced by the model without nonlocal terms and periodic boundary conditions are studied first. Then, Turing patterns induced by the nonlocal interaction (see Banerjee et al. (2018) [1]) in the two dimensional space are explored along with the effects of the nonlocal interaction range on the resulting patterns under proper parametric restrictions. The Turing bifurcation conditions for the nonlocal model are derived analytically and bifurcation scenario of stationary hotspot pattern generated from the homogeneous steady-state are studied in detail, both analytically and numerically. Also, conversion of periodic and aperiodic solutions exhibited by the local model into stationary Turing pattern as an effect of the nonlocal interaction term is also explored. The resulting patterns are stationary when the range of nonlocal interactions are significantly large.
\end{abstract}

(c) 2020 Published by Elsevier B.V.

\section{Introduction}

Reaction-diffusion systems provide an appropriate tool to study spatio-temporal pattern formation in various ecological processes. Patterns induced by diffusion, also called dissipative or Turing patterns arise due to the instability of a homogeneous in space solution $[2,10]$ resulting in the appearance of spots, stripes, and other structures. Other mechanisms of pattern formation lead to the emergence of traveling waves, target and spiral patterns, periodic traveling waves [9,29], interacting spiral chaos, spatio-temporal chaos, etc. $[6,7,16,19,21]$. Let us recall that cold spot patterns refer to the regions where the population density is lower than around them, whereas hot spot patterns correspond to the regions with a higher population density. Labyrinthine pattern is represented by the interlaced bands of high and low population densities forming stripes. These and other stationary patterns are formed by interacting populations due to the process of self-organization. Pattern formation in ecology is intensively studied in the case of plankton patchiness [14,22], semiarid vegetation patterns [13], invasion by exotic species [17,25] and so on.

A conventional assumption in ecological model is that individuals in the population consume their resources locally, that is at the spatial point where they are located at a particular time. In a more realistic scenario of nonlocal consumption of

\footnotetext{
* Corresponding author.

E-mail addresses: nayana@imsc.ac.in (N. Mukherjee), volpert@math.univ-lyon1.fr (V. Volpert).
} 
resources, they can search food in some area around their average location. Global consumption of resources, on the other hand, corresponds to the situation where the population can consume resource in the whole area of its spreading. The models describing nonlocal and global consumption of resources include integro-differential terms in the reaction-diffusion equations [12,24]. They allow the description of the emergence and evolution of biological species and, in a more general context, of the process of speciation $[11,26]$. These problems are studied for single species models and for the competition models including two or more species $[11,18,27,28]$.

The local spatio-temporal prey-predator model with Rosenzweig-McArthur type reaction kinetics [24] does not support the emergence of Turing patterns. However, due to the introduction of nonlocal terms, Turing patterns appear under proper assumptions on parameters [4]. Existence of non-Turing patterns like traveling wave, modulated traveling wave, oscillatory pattern and spatio-temporal chaos are also observed for the nonlocal model. Other non-Turing patterns are observed for the modified Lotka-Volterra reaction kinetics describing the effects of nonlocal consumption of resources [4,5].

In our previous work, we have considered a model of nonlocal consumption of resources by prey with the bistable reaction kinetics in the absence of predator and in the presence of a specialist predator following Holling type-II functional response [1]. Formation of Turing patterns and evolution of other spatio-temporal patterns are studied in one spatial dimension. In this work, we are interested in two-dimensional Turing patterns described by the same nonlocal model. The influence of the range of nonlocal interaction on stationary patterns are also explored in this work. The paper is organized as follows. The description of the model and the derivation of conditions of the Turing instability are given in Section 2. Simulation results are discussed in Section 3. Main outcomes of this investigation are summarized in the concluding Section 4.

\section{The model}

Derivation of the single species population model with additive Allee effect from the classical single species population models is discussed in Petrovskii et al. [20]. Allee effects are generally defined as a decline in individual fitness at low population size or density, due to various reasons like mating procedure, predation, environmental modification, etc. that can result in critical population thresholds below which populations are driven to extinction. Hence, including Allee effect in the model makes the modelling approach much more realistic specially at low population density. The concept of multiplicative Allee effect is described in Amarasekare [3], Courchamp et al. [8]. When the multiplicative Allee effect is included in the growth equation of the single species population, it accounts for two significant feedback effects: positive feedback due to cooperation at low population density and negative feedback arising through the competition for limited resources at high population density [15]. As an alternative formulation of the same, Petrovskii et al. [20] proposed an additive form of the per capita growth rate function which includes population growth due to the reproduction and density dependent enhanced mortality rate at low population density due to Allee effect. Details of the derivation of mathematical formulation for growth rate affected by Allee effect can be found in Banerjee et al. [1]. We now briefly describe the temporal model and the spatio-temporal model in two spatial dimensions, followed by the spatio-temporal model with nonlocal interaction in the upcoming subsections.

\subsection{Temporal model}

Using the growth function including sexual reproduction for prey population and its density dependent mortality rate in form of additive Allee effect and Holling type II functional response of the specialist predator we get the temporal model

$$
\begin{aligned}
& \frac{d u}{d t}=a u^{2}(b-u)-\sigma_{1} u-\frac{\alpha u v}{\kappa+u}, \\
& \frac{d v}{d t}=\frac{\beta u v}{\kappa+u}-\sigma_{2} v,
\end{aligned}
$$

subjected to non-negative initial conditions. Here $a$ is the intrinsic growth rate, $b$ is the environmental carrying capacity and $\sigma_{1}$ is the density independent natural death rate of the prey population. Also $\alpha$ is the rate of consumption of prey by an individual predator, $\kappa$ is the half-saturation constant, $\beta$ is the rate of conversion of prey to predator biomass due to new born predator individuals and $\sigma_{2}$ is the natural mortality rate of the predator. Furthermore, $\beta / \alpha$ is the conversion efficiency with the value between 0 and 1 , consequently $0<\beta<\alpha$. The reproduction of prey is proportional to the second power of the population density specific for sexual reproduction [20]. It is to be noted that for the prey population, the reproduction rate is proportional to the product of $u_{1}, u_{2}, R$, where $u_{1}$ is the density of females, $u_{2}$ of males, and $R$ available resources. In particular, if there are no resources, the reproduction is impossible. Next, available resources can be determined as carrying capacity minus consumed resources, while consumed resources are proportional to the sum of the densities of males and females: $R=K-B\left(u_{1}+u_{2}\right)$. Assuming that the densities of males and females are approximately equal to each other i.e $u_{1}=u_{2}=u$, we obtain the growth term to be $u^{2}(K-2 B u)=a u^{2}(b-u)$ where $a=2 B$ and $b=K / 2 B$. Thus, the term $-u^{3}$ means that there are in fact two different competitions, one of them for resources and another one for sexual partners.

In the absence of predator $(\alpha=0)$ the prey population shows bistable dynamics. The equilibrium points of the temporal model (2.1) and (2.2) are total extinction state $(0,0)$ which is asymptotically stable, predator free axial equilibrium points $\left(u_{1}, 0\right) \equiv\left(\frac{b-\left(b^{2}-\frac{4 \sigma_{1}}{a}\right)^{\frac{1}{2}}}{2}, 0\right)$ and $\left(u_{2}, 0\right) \equiv\left(\frac{b+\left(b^{2}-\frac{4 \sigma_{1}}{a}\right)^{\frac{1}{2}}}{2}, 0\right)$, which are feasible for $b^{2}>\frac{4 \sigma_{1}}{a}$. We will discuss the transcritical 

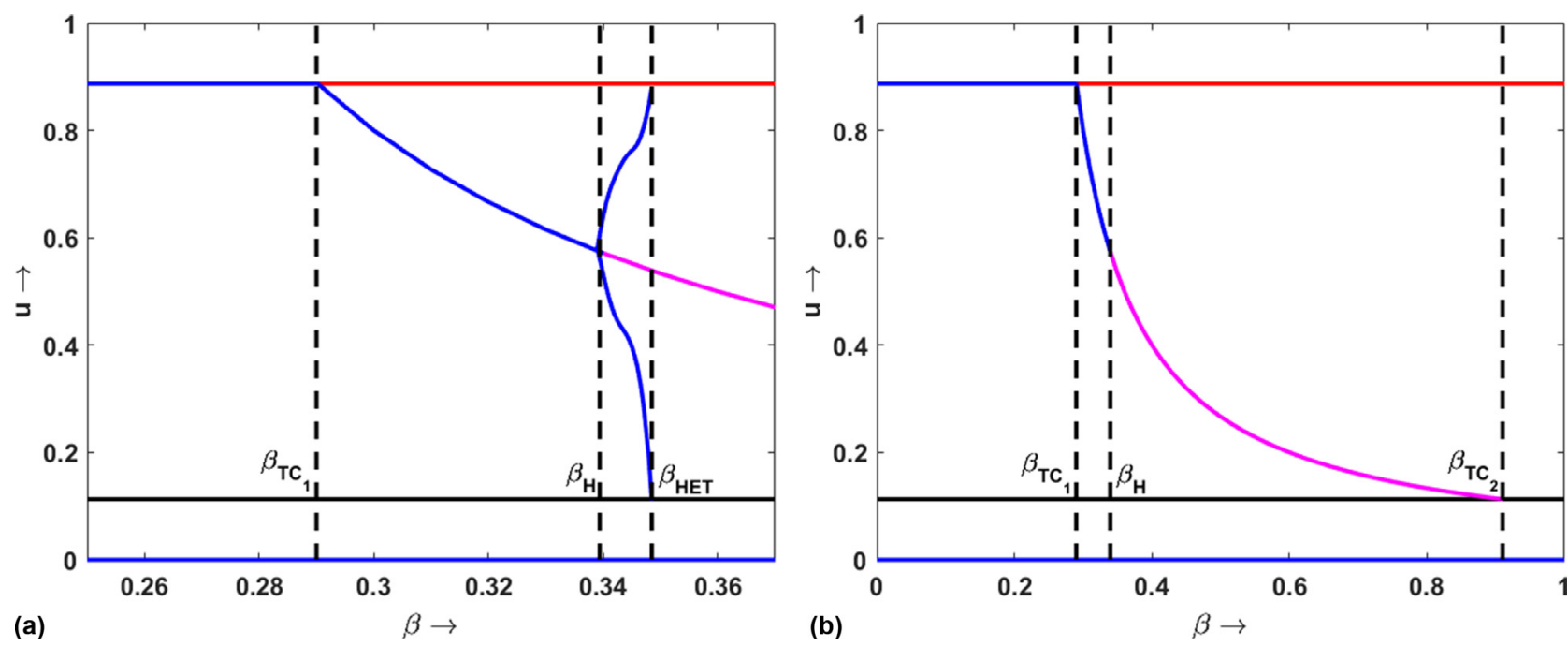

Fig. 1. Bifurcation diagram of temporal model (2.1) and (2.2) with respect to $\beta$. The other parameter values are taken to be $a=1, b=1, \sigma_{1}=0.1, \alpha=$ $0.4, \kappa=0.4, \sigma_{2}=0.2$; (a) $\beta \in(0,1)$ (b) zoomed version of (a) where $\beta \in(0.25,0.37)$.

bifurcation scenario with the help of a numerical example as shown in Fig. 1. The interior equilibrium point is denoted by $\left(u_{*}, v_{*}\right)$ whose components are

$$
u_{*}=\frac{\kappa \sigma_{2}}{\beta-\sigma_{2}}, \quad v_{*}=\left(a u_{*}\left(b-u_{*}\right)-\sigma_{1}\right)\left(\kappa+u_{*}\right) / \alpha
$$

The interior equilibrium point is feasible under the parametric restrictions $\beta>\sigma_{2}$ as well as $a b u_{*}>a u_{*}^{2}+\sigma_{1}$. The Jacobian matrix of the model (2.1) and (2.2) evaluated at $\left(u_{*}, v_{*}\right)$ is given by

$$
H=\left[\begin{array}{cc}
a b u_{*}-2 a u_{*}^{2}+\frac{\alpha u_{*} v_{*}}{\left(\kappa+u_{*}\right)^{2}} & -\frac{\alpha u_{*}}{\kappa+u_{*}} \\
\frac{\beta \kappa v_{*}}{\left(\kappa+u_{*}\right)^{2}} & 0
\end{array}\right] \equiv\left[\begin{array}{cc}
a_{11} & a_{12} \\
a_{21} & 0
\end{array}\right] .
$$

Hence, the local asymptotic stability condition for $\left(u_{*}, v_{*}\right)$ is given by the following condition:

$$
a b u_{*}-2 a u_{*}^{2}+\frac{\alpha u_{*} v_{*}}{\left(\kappa+u_{*}\right)^{2}}<0
$$

The condition for Hopf bifurcation through which the interior equilibrium point $\left(u_{*}, v_{*}\right)$ becomes unstable and gives periodic solution is determined by the equality:

$$
a b u_{*}-2 a u_{*}^{2}+\frac{\alpha u_{*} v_{*}}{\left(\kappa+u_{*}\right)^{2}}=0
$$

From the bifurcation diagram (Fig. 1) it is clear that the dynamics of system (2.1) and (2.2) changes as $\beta$ increases from 0 to 1. Till $\beta<\beta_{T C_{1}}=0.29$, the first transcritical bifurcation threshold, the axial equilibrium point $\left(u_{2}, 0\right) \equiv(0.887,0)$ is stable and at $\beta_{T C_{1}}=0.29,\left(u_{*}, v_{*}\right)$ bifurcates from $\left(u_{2}, 0\right)$. For $\beta>\beta_{T C_{1}},\left(u_{2}, 0\right)$ is saddle point and $\left(u_{*}, v_{*}\right)$ is asymptotically stable whenever $\beta_{T C_{1}}<\beta<\beta_{H}$. Also, $\left(u_{*}, v_{*}\right)$ loses its stability at the Hopf bifurcation threshold $\beta_{H}=0.3394$ and becomes unstable till the second transcritical bifurcation threshold $\beta=\beta_{T C_{2}}=0.91$. At $\beta=\beta_{T C_{2}},\left(u_{*}, v_{*}\right)$ merges with $\left(u_{1}, 0\right) \equiv(0.113,0)$. The limit cycle generated through Hopf bifurcation is stable, as the Hopf bifurcation is supercritical. As values of $\beta>\beta_{H}$ are considered, stable limit cycle enlarges in size gradually and disappears through a global bifurcation at $\beta_{H E T}=0.348$ making a heteroclinic connection between $\left(u_{1}, 0\right)$ and $\left(u_{2}, 0\right)$ equilibrium points. The two species coexist only for $\beta_{T C_{1}}<\beta<\beta_{H E T}$ [23]. This bifurcation scenario of the temporal model (2.1) and (2.2) is necessary to be discussed to better understand the dynamics of the spatio-temporal extension of the model in two spatial dimensions which is discussed next.

\subsection{Spatio-temporal local model in two dimensions}

Consider a rectangular bounded domain $\Omega \subset \mathbb{R}^{2}$ with closed boundary $\partial \Omega$. Let $u(x, y, t)$ and $v(x, y, t)$ be the nondimensional population densities at position $(x, y) \in \Omega$ and time $t$ for the prey and predator populations respectively. In terms of the dimensionless variables, the spatio-temporal model corresponding to the temporal model (2.1) and (2.2) is described by the following reaction-diffusion system of equations:

$$
\frac{\partial u}{\partial t}=d_{1}\left(\frac{\partial^{2} u}{\partial x^{2}}+\frac{\partial^{2} u}{\partial y^{2}}\right)+a u^{2}(b-u)-\sigma_{1} u-\frac{\alpha u v}{\kappa+u},
$$




$$
\frac{\partial v}{\partial t}=d_{2}\left(\frac{\partial^{2} v}{\partial x^{2}}+\frac{\partial^{2} v}{\partial y^{2}}\right)+\frac{\beta u v}{\kappa+u}-\sigma_{2} v
$$

subjected to non-negative initial condition and periodic boundary conditions. Here $d_{1}$ is the diffusion coefficient of prey and $d_{2}$ is that of predator. The interior equilibrium point $\left(u_{*}, v_{*}\right)$ of $(2.1)$ and (2.2) is a homogeneous steady-state of the spatiotemporal model. It is important to mention here that the spatio-temporal model (2.5) and (2.6) does not describe stationary Turing patterns since one of the diagonal elements of $H$ is zero. The stability conditions in the absence of diffusion are $a_{11}<0$ and $a_{12} a_{21}<0$, as discussed before. We perturb the homogeneous steady state $\left(u_{*}, v_{*}\right)$ of system (2.5) and (2.6) as follows:

$$
\begin{aligned}
& u(x, t)=u_{*}+C_{1} e^{\lambda t+i\left(k_{x} x+k_{y} y\right)}, \\
& v(x, t)=v_{*}+C_{2} e^{\lambda t+i\left(k_{x} x+k_{y} y\right)},
\end{aligned}
$$

where $0<C_{1}, C_{2} \ll 1, \lambda$ is the growth rate of perturbations, $\mathbf{k}=\left(k_{x}, k_{y}\right)$ is the wave number vector and $k=|\mathbf{k}|$ is the wave number. Substituting (2.7) into (2.5) and (2.6), the characteristic equation for the growth rate $\lambda$ is found from the equality $\operatorname{Det}\left(\mathbf{H}_{1}\right)=0$, where

$$
\mathbf{H}_{1}=\left(\begin{array}{cc}
a_{11}-d_{1} k^{2}-\lambda & a_{12} \\
a_{21} & -d_{2} k^{2}-\lambda
\end{array}\right) .
$$

The explicit expressions for $a_{11}, a_{12}$ and $a_{21}$ are given in the previous subsection. The characteristic equation is given by the equality:

$$
\lambda^{2}-\lambda\left(a_{11}-k^{2}\left(d_{1}+d_{2}\right)\right)+h\left(k^{2}\right)=0,
$$

where

$$
h\left(k^{2}\right)=d_{1} d_{2} k^{4}-d_{2} a_{11} k^{2}-a_{12} a_{21} .
$$

Equating the derivative $\frac{d h\left(k^{2}\right)}{d\left(k^{2}\right)}$ of $h\left(k^{2}\right)$ with respect to $k^{2}$ to zero at $k=k_{T}$, which is the critical wave number for the Turing instability, gives

$$
k_{T}^{2}=\frac{a_{11}}{2},
$$

which implies that $a_{11}$ has to be positive to maintain the positivity of $k_{T}^{2}$. However, from the conditions of Turing instability, we know that $a_{11}<0$ is required for the stability of homogeneous steady state under temporal perturbations. Thus, Turing instability conditions cannot be satisfied for the local spatio-temporal model (2.5) and (2.6). Nevertheless, the model gives variety of dynamic patterns which are discussed in the section of simulation results. Next, we consider the nonlocal consumption of resources by prey in the spatio-temporal model.

\subsection{Nonlocal model in two spatial dimensions}

Under the assumption that prey can move from one location to another one to access the resources, the spatio-temporal model in two spatial dimensions with nonlocal consumption of resources has the form

$$
\begin{aligned}
& \frac{\partial u}{\partial t}=d_{1}\left(\frac{\partial^{2} u}{\partial x^{2}}+\frac{\partial^{2} u}{\partial y^{2}}\right)+a u^{2}(b-J(u))-\sigma_{1} u-\frac{\alpha u v}{\kappa+u}, \\
& \frac{\partial v}{\partial t}=d_{2}\left(\frac{\partial^{2} v}{\partial x^{2}}+\frac{\partial^{2} v}{\partial y^{2}}\right)+\frac{\beta u v}{\kappa+u}-\sigma_{2} v,
\end{aligned}
$$

subjected to non-negative initial condition and the periodic boundary condition. The nonlocal interaction term is given by the integral

$$
\begin{aligned}
& J(u)=\int_{-\infty}^{\infty} \int_{-\infty}^{\infty} K_{\delta_{1}, \delta_{2}}(p-x, q-y) u(t, p, q) d p d q, \\
& K_{\delta_{1}, \delta_{2}}\left(z_{1}, z_{2}\right)=\left\{\begin{array}{cc}
\frac{1}{4 \delta_{1} \delta_{2}}, & |x| \leq \delta_{1},|y| \leq \delta_{2} \\
0, & \text { otherwise }
\end{array}\right.
\end{aligned}
$$

Now, we will analyze stability of the homogeneous steady-state $\left(u_{*}, v_{*}\right)$ for the nonlocal model (2.11) and (2.12). We consider the perturbation around it in the form

$$
u(x, t)=u_{*}+\epsilon_{1} e^{\lambda t+i\left(k_{x} x+k_{y} y\right)}, \quad v(x, t)=v_{*}+\epsilon_{2} e^{\lambda t+i\left(k_{x} x+k_{y} y\right)}, \quad\left|\epsilon_{1}\right|,\left|\epsilon_{2}\right| \ll 1 .
$$


The characteristic equation writes as $|J-\lambda I|=0$, where

$$
J=\left[\begin{array}{cc}
a_{1}-a u_{*}^{2} \frac{\sin \left(k_{x} \delta_{1}\right)}{k_{x} \delta_{1}} \frac{\sin \left(k_{y} \delta_{2}\right)}{k_{y} \delta_{2}}-d_{1}\left(k_{x}^{2}+k_{y}^{2}\right) & -a_{2} \\
b_{1} & -d_{2}\left(k_{x}^{2}+k_{y}^{2}\right)
\end{array}\right]
$$

and

$$
a_{1}=a b u_{*}-a u_{*}^{2}+\frac{\alpha u_{*} v_{*}}{\left(\kappa+u_{*}\right)^{2}}, a_{2}=\frac{\alpha u_{*}}{\kappa+u_{*}}, b_{1}=\frac{\beta \kappa v_{*}}{\left(\kappa+u_{*}\right)^{2}}
$$

Therefore, the characteristic equation is of the form

$$
\lambda^{2}-\Gamma(k, M) \lambda+\Delta(k, M)=0,
$$

where

$$
\begin{aligned}
& \Gamma\left(k_{x}, k_{y}, \delta_{1}, \delta_{2}\right)=a_{1}-a u_{*}^{2} \frac{\sin \left(k_{x} \delta_{1}\right)}{k_{x} \delta_{1}} \frac{\sin \left(k_{y} \delta_{2}\right)}{k_{y} \delta_{2}}-\left(d_{1}+d_{2}\right)\left(k_{x}^{2}+k_{y}^{2}\right), \\
& \Delta\left(k_{x}, k_{y}, \delta_{1}, \delta_{2}\right)=\left(a u_{*}^{2} \frac{\sin \left(k_{x} \delta_{1}\right)}{k_{x} \delta_{1}} \frac{\sin \left(k_{y} \delta_{2}\right)}{k_{y} \delta_{2}}-a_{1}+d_{1}\left(k_{x}^{2}+k_{y}^{2}\right)\right) d_{2}\left(k_{x}^{2}+k_{y}^{2}\right)+a_{2} b_{1} .
\end{aligned}
$$

We first assume that the conditions for local asymptotic stability of the interior equilibrium point of the corresponding temporal model (2.1) and (2.2) are satisfied (condition (2.3) holds). The homogeneous coexistence steady-state of the local spatio-temporal model (2.5) and (2.6) does not lose its stability under spatio-temporal perturbations. The homogeneous steady-state of the nonlocal model (2.11) and (2.12) is stable under space dependent perturbations if the following two conditions are satisfied:

$$
\Gamma\left(k_{x}, k_{y}, \delta_{1}, \delta_{2}\right)<0, \Delta\left(k_{x}, k_{y}, \delta_{1}, \delta_{2}\right)>0,
$$

and unstable if

$$
\Gamma\left(k_{x}, k_{y}, \delta_{1}, \delta_{2}\right)<0, \Delta\left(k_{x}, k_{y}, \delta_{1}, \delta_{2}\right)<0,
$$

for any combination of values of $k_{x}, k_{y}, \delta_{1}, \delta_{2}>0$. Here we have reversed the direction of second inequality to get the Turing instability condition. In order to simplify the forthcoming calculation we consider $\delta_{1}=\delta_{2}$ and fix them to some positive value. The critical wavenumber $\left(k_{x}, k_{y}\right)$ and the corresponding Turing bifurcation threshold in terms of $d$ can be obtained as appropriate solution of the following three equations:

$$
\Delta\left(k_{x}, k_{y}, \delta_{1}, \delta_{2}\right)=0, \quad \frac{\partial}{\partial k_{x}} \Delta\left(k_{x}, k_{y}, \delta_{1}, \delta_{2}\right)=0, \quad \frac{\partial}{\partial k_{y}} \Delta\left(k_{x}, k_{y}, \delta_{1}, \delta_{2}\right)=0 .
$$

Solving equation $\Delta\left(k_{x}, k_{y}, \delta_{1}, \delta_{2}\right)=0$ with respect to $d$, we get

$$
d_{2}\left(k_{x}, k_{y}\right)=\frac{a_{2} b_{1}}{\left(k_{x}^{2}+k_{y}^{2}\right)\left(a_{1}-d_{1}\left(k_{x}^{2}+k_{y}^{2}\right)-a u_{*}^{2} \frac{\sin k_{x} \delta_{1}}{k_{x} \delta_{1}} \frac{\sin k_{y} \delta_{2}}{k_{y} \delta_{2}}\right)} .
$$

Substituting this expression for $d_{2}$ into the second and third conditions of (2.20) leads to two transcendental equations in terms of two unknowns $k_{x}$ and $k_{y}$ which cannot be solved explicitly. We numerically calculate the positive zeros of two transcendental equations over a considerable range of values of $k_{x}$ and $k_{y}$. Substituting these solutions in (2.21), we find the minimal positive value of $d_{2}$ which gives the Turing threshold. If we are unable to find any feasible solution for the transcendental equations and/or the values of $d_{2}$ are negative, then the Turing bifurcation is not possible for the chosen value of $\delta_{1}=\delta_{2}$ and other chosen parameter values. Before all this we need to find a feasible range of $k_{x}$ and $k_{y}$ values in the $k_{x}-k_{y}$ plane for which $\Delta<0$ and $\operatorname{Re}(\lambda)>0$ such that the Turing bifurcation threshold is already crossed. We will illustrate this approach with a numerical example in the upcoming section.

\subsection{Bifurcation of stationary pattern from homogeneous steady-state}

Here we discuss the bifurcation of stationary with respect to time patterns from homogeneous steady-state with the help of perturbation theory. Let $\alpha$ be a bifurcation parameter. For all values of this parameter, the constant $u_{0}=\frac{\kappa \sigma_{2}}{\beta-\sigma_{2}}, v_{0}=$ $\left(a u_{*}\left(b-u_{*}\right)-\sigma_{1}\right)\left(\kappa+u_{*}\right) / \alpha$ is a solution of this problem. When $\alpha$ crosses a critical value, a simple negative real eigenvalue of the linearized problem crosses the origin and a bifurcation occurs. Thus the perturbation of $\alpha$ can be considered as

$$
\alpha=\alpha_{0}+\epsilon \alpha_{1}+\epsilon^{2} \alpha_{2}+\epsilon^{3} \alpha_{3}+\ldots
$$

In order to study this bifurcation, we look for the solution in the form of the expansion based on that of $\alpha$ to be

$$
\begin{aligned}
& u=u_{0}+\epsilon u_{1}+\epsilon^{2} u_{2}+\epsilon^{3} u_{3}+\ldots, \\
& v=v_{0}+\epsilon v_{1}+\epsilon^{2} v_{2}+\epsilon^{3} v_{3}+\ldots
\end{aligned}
$$


Substituting these expansions into the following equations,

$$
\begin{aligned}
& \left(\frac{\partial^{2} u}{\partial x^{2}}+\frac{\partial^{2} u}{\partial y^{2}}\right)+a u^{2}(b-J(u))-\sigma_{1} u-\frac{\alpha u v}{\kappa+u}=0 \\
& d\left(\frac{\partial^{2} v}{\partial x^{2}}+\frac{\partial^{2} v}{\partial y^{2}}\right)+\frac{\beta u v}{\kappa+u}-\sigma_{2} v=0
\end{aligned}
$$

denoting $\left(\frac{\partial^{2} u}{\partial x^{2}}+\frac{\partial^{2} u}{\partial y^{2}}\right) \equiv \Delta$ and equating the terms with the first power of $\epsilon$, we get

$$
\left(\begin{array}{c}
\Delta u_{1} \\
\Delta v_{1}
\end{array}\right)+\left(\begin{array}{c}
\left(\alpha_{0} A_{1}+B_{1}\right) u_{1}-a u_{0}^{3} J\left(u_{1}\right)-\alpha_{0} A_{2} v_{1} \\
b_{1} u_{1}
\end{array}\right)=\left(\begin{array}{l}
0 \\
0
\end{array}\right)
$$

where

$$
A_{1}=\frac{u_{0} v_{0}}{\left(\kappa+u_{0}\right)^{2}}, B_{1}=a b u_{0}-a u_{0}^{2}, A_{2}=\frac{u_{0}}{\kappa+u_{0}}, \quad b_{1}=\frac{\beta \kappa v_{0}}{\left(\kappa+u_{0}\right)^{2}} .
$$

This is the corresponding eigenvalue problem of (2.22a) and (2.22b) with 0 eigenvalue. Thus the eigenfunctions can be chosen as

$$
u_{1}=\cos \left(m_{1} x\right) \cos \left(m_{2} y\right), v_{1}=\cos \left(m_{1} x\right) \cos \left(m_{2} y\right),
$$

where $m_{1}, m_{2} \neq 0$ are integers. Now equating the terms with $\epsilon^{2}$ we get,

$$
\left(\begin{array}{c}
\Delta u_{2} \\
\Delta v_{2}
\end{array}\right)+\left(\begin{array}{c}
\left(\alpha_{0} A_{1}+B_{1}\right) u_{2}-a u_{0}^{3} J\left(u_{2}\right)-\alpha_{0} A_{2} v_{2} \\
b_{1} u_{2}
\end{array}\right)=\left(\begin{array}{c}
f_{1} \\
0
\end{array}\right)
$$

where

$$
f_{1}=-\alpha_{1} A_{1} u_{1}-a u_{0}^{2} u_{1} J\left(u_{1}\right)+A_{2} \alpha_{1} v_{1} .
$$

In order to obtain solvability conditions for problem (2.26), let us note that problem (2.23) is self-adjoint since the kernel $K$ is an even function. Hence (2.26) is solvable if

$$
\int_{-\delta_{1}}^{\delta_{1}} \int_{-\delta_{2}}^{\delta_{2}} f_{1}(x, y) u_{1}(x, y) d x d y=0 .
$$

Solving for $\alpha_{1}$ we get

$$
\alpha_{1}=\frac{\int_{-\delta_{1}}^{\delta_{1}} \int_{-\delta_{2}}^{\delta_{2}} \alpha_{0} A_{2} v_{1} d x d y-\int_{-\delta_{1}}^{\delta_{1}} \int_{-\delta_{2}}^{\delta_{2}}\left(\alpha_{0} A_{1}+B\right) u_{1} d x d y}{\int_{-\delta_{1}}^{\delta_{1}} \int_{-\delta_{2}}^{\delta_{2}} A_{2} v_{1} d x d y-\int_{-\delta_{1}}^{\delta_{1}} \int_{-\delta_{2}}^{\delta_{2}} A_{1} u_{1} d x d y}
$$

If $\alpha_{1} \neq 0$, then from sign of $\alpha_{1}$ we get the type of bifurcation. If $\alpha_{1}<0$ then it is subcritical bifurcation. If $\alpha_{1}>0$ then it is a supercritical bifurcation. In case if $\alpha_{1}=0$ then we equate the terms of $\epsilon^{3}$ and using solvability conditions, we find $\alpha_{2}$ based on sign of which we can determine the type of bifurcation. The $\epsilon^{3}$ terms are of the form

$$
\left(\begin{array}{c}
\Delta u_{3} \\
\Delta v_{3}
\end{array}\right)+\left(\begin{array}{c}
\left(\alpha_{0} A_{1}+B_{1}\right) u_{3}-a u_{0}^{3} J\left(u_{3}\right)-\alpha_{0} A_{2} v_{3} \\
b_{1} u_{3}
\end{array}\right)=\left(\begin{array}{c}
f_{2} \\
0
\end{array}\right)
$$

where

$$
f_{2}=-\alpha_{2} A_{1} u_{1}-a u_{0}^{2}\left(u_{1} J\left(u_{2}\right)+u_{2} J\left(u_{1}\right)\right)+A_{2} \alpha_{2} v_{1},
$$

taking into account $\alpha_{1}=0$. In order to obtain solvability conditions for problem (2.26), let us note that problem (2.23) is self-adjoint since the kernel $K$ is an even function. Hence (2.26) is solvable if

$$
\int_{-\delta_{1}}^{\delta_{1}} \int_{-\delta_{2}}^{\delta_{2}} f_{2}(x, y) u_{1}(x, y) d x d y=0 .
$$

Solving for $\alpha_{2}$ we get,

$$
\alpha_{2}=\frac{\int_{-\delta_{1}}^{\delta_{1}} \int_{-\delta_{2}}^{\delta_{2}} a u_{0}^{2} u_{1} J\left(u_{2}\right) d x d y+\int_{-\delta_{1}}^{\delta_{1}} \int_{-\delta_{2}}^{\delta_{2}} a u_{0}^{2} u_{2} J\left(u_{1}\right) d x d y}{\int_{-\delta_{1}}^{\delta_{1}} \int_{-\delta_{2}}^{\delta_{2}} A_{2} v_{1} d x d y-\int_{-\delta_{1}}^{\delta_{1}} \int_{-\delta_{2}}^{\delta_{2}} A_{1} u_{1} d x d y}
$$

Similarly, as discussed above, if $\alpha_{2} \neq 0$, then from sign of $\alpha_{2}$ we get the type of bifurcation. If $\alpha_{2}<0$ then it is subcritical bifurcation. If $\alpha_{2}>0$ then it is a supercritical bifurcation. In case if $\alpha_{2}=0$ then we equate the terms of $\epsilon^{4}$ and using solvability conditions, we find $\alpha_{3}$ based on sign of which we can determine the type of bifurcation. Now we consider some numerical results to show the bifurcation actually happening in the next section. 

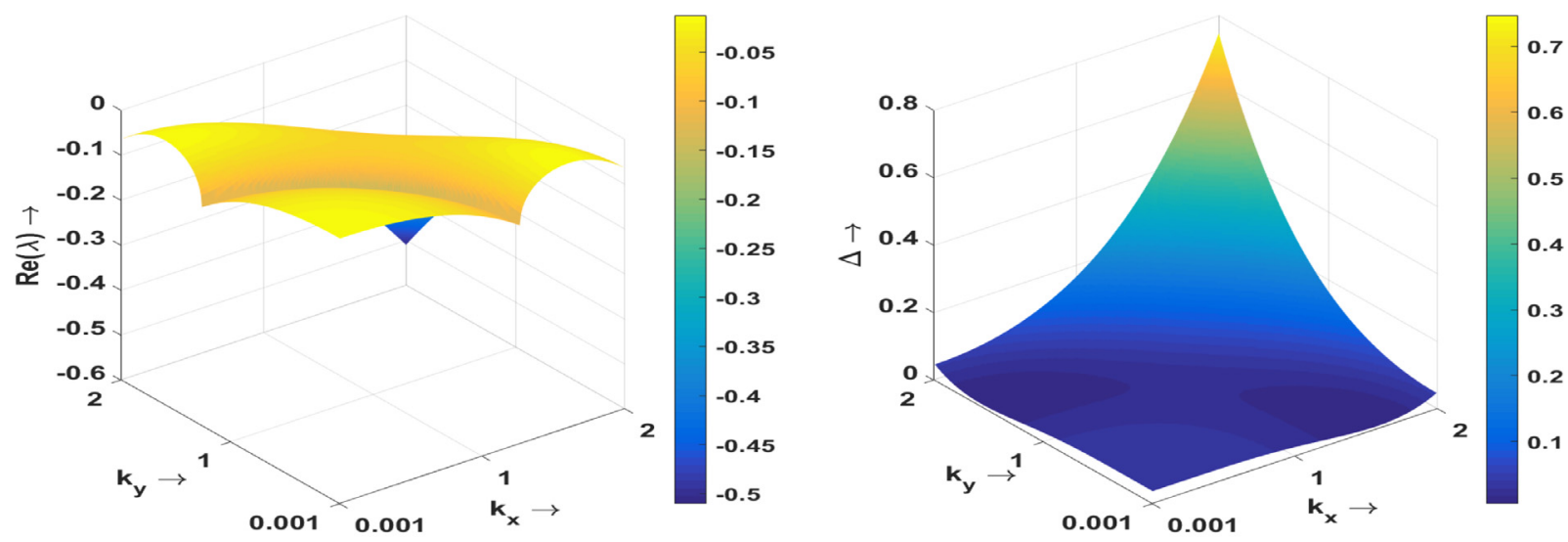

(a)
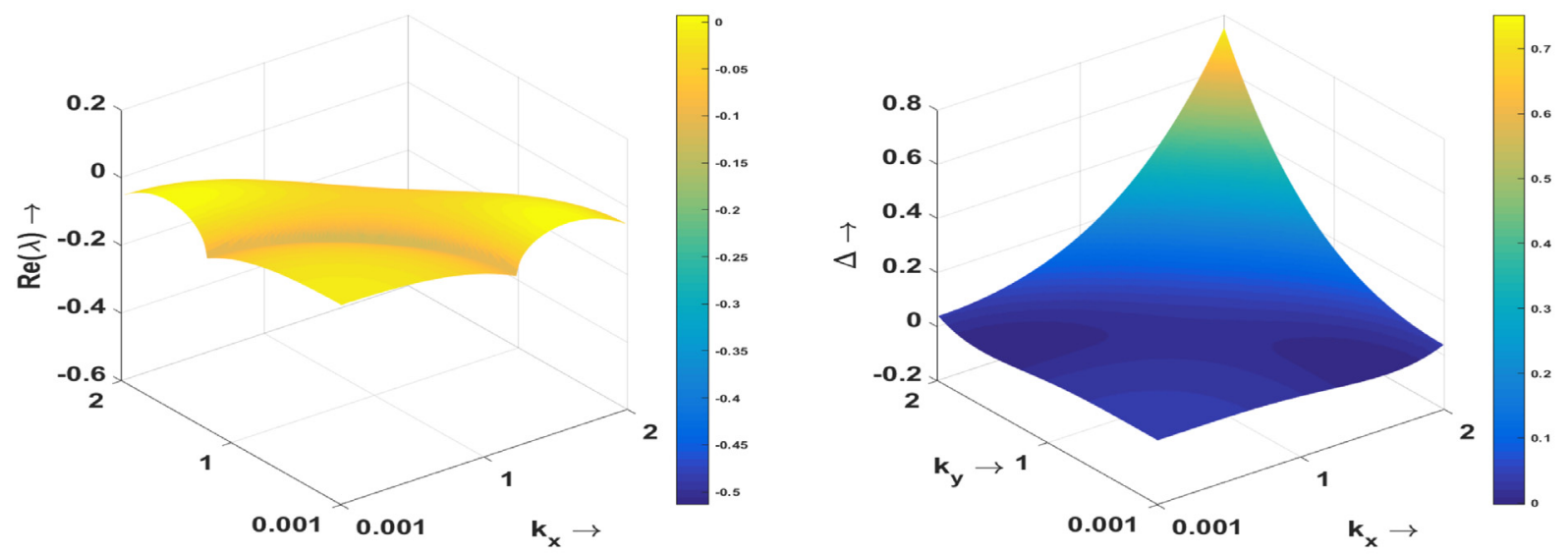

(b)

Fig. 2. Surface plots of $\operatorname{Re}(\lambda)$ (left) and $\Delta$ (right) for $k_{x}$ and $k_{y}$ ranging from 0.001 to 2 and (a) $d=0.189, \delta_{1}=\delta_{2}=1.9$; (b) $d=0.189$, $\delta_{1}=\delta_{2}=2$ and other parameter values as mentioned in the text.

\section{Simulation results}

Here we illustrate that, one cannot find Turing patterns without the inclusion of nonlocal interaction term into the local model (2.5) and (2.6). The numerical simulations are performed using five-point stencil finite difference scheme for the diffusion part and forward Euler method for the temporal part of both the local and nonlocal model with periodic boundary conditions on a $100 \times 100$ mesh with $\Delta x=\Delta y=1$ and $\Delta t=0.01$. The results have been verified taking other values of $\Delta x, \Delta y$ and $\Delta t$, and no significant changes have been observed. A small random perturbation to the homogeneous steady state of the system is taken to be the initial condition for all the numerical simulations which is of the form

$$
\begin{gathered}
u\left(x, y, t_{0}\right)=u_{*}+0.001 * \text { rand, } \\
v\left(x, y, t_{0}\right)=v_{*}+0.001 * \text { rand, }
\end{gathered}
$$

where $t_{0}$ is the initial time and rand is the MATLAB function which takes up positive no.s between 0 and 1 . First we demonstrate the Turing instability conditions and generation of Turing patterns in two-dimensional space with a numerical example.

\subsection{Numerical example of two dimensional Turing patterns}

Let us consider the parameter set $a=1, b=1, \sigma_{1}=0.1, \alpha=0.335, \kappa=0.4, \beta=0.335, \sigma_{2}=0.2 d_{1}=0.1 d_{2}=$ 0.189. Keeping the parameter range similar as in Banerjee et al. [1], we carry out analysis in two spatial dimensions. Instead of a single wavenumber $k$, we now consider two wavenumbers, $k_{x}$ along $x$ direction and $k_{y}$ along $y$ direction as the 


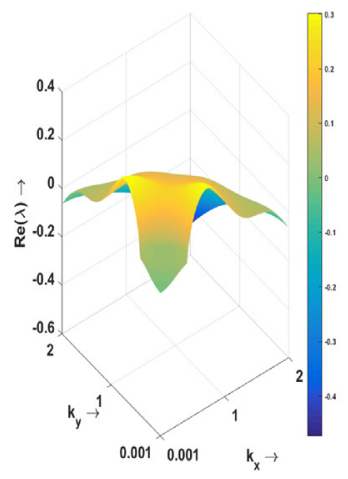

(a)

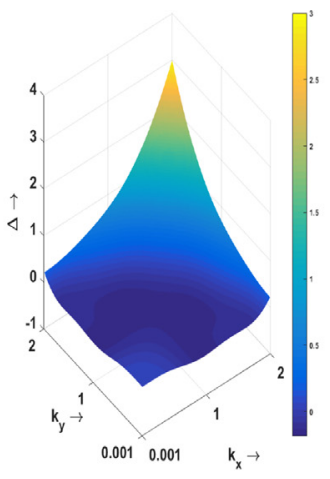

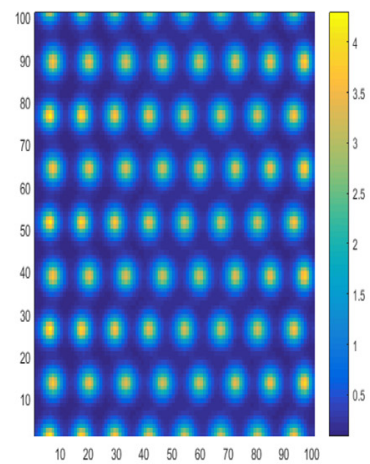

(b)

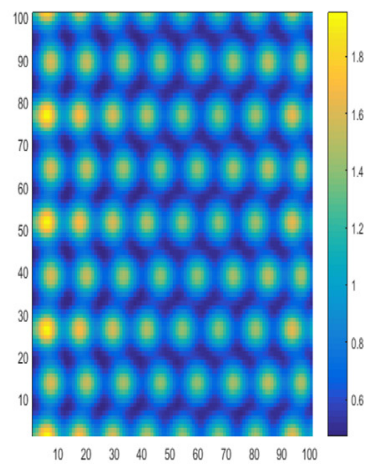

(c)

Fig. 3. (a) Surface plots of $\operatorname{Re}(\lambda)$ (left) and $\Delta$ (right) for $k_{x}$ and $k_{y}$ ranging from 0.001 to 2 and $d=0.8, \delta_{1}=\delta_{2}=6$; (b) Stationary patterns for the prey population produced by the nonlocal model (2.11) and (2.12) for the corresponding parameter set; (c) Stationary pattern for the corresponding predator population; other parameter values are mentioned in the text.

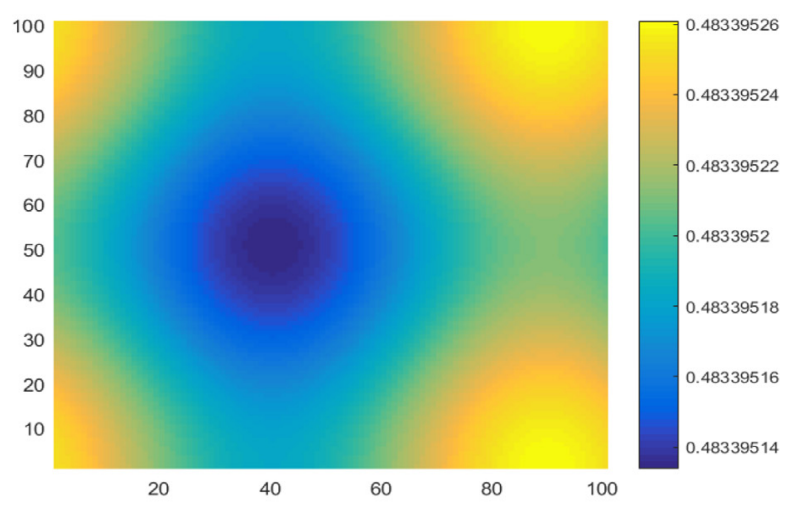

(a)

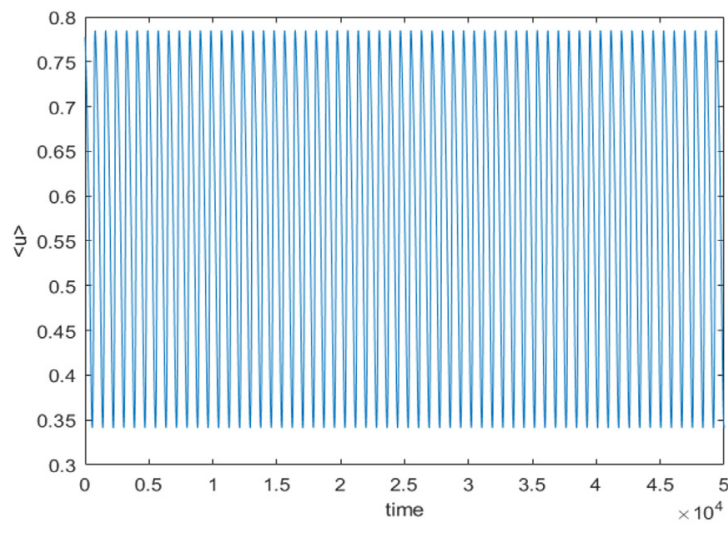

(b)

Fig. 4. Patterns observed for the prey population for $\beta=0.3445$ : (a) snapshot of oscillatory with respect to time solution exhibited by the local model where the pattern is almost homogeneous in space but oscillatory in time; (b) spatial average of the prey population density plotted with respect to time.

space considered is two-dimensional. For the local model (2.5) and (2.6), no matter what parameter set we choose, we can never find an area in $k_{x}-k_{y}$ plane for which $\operatorname{Re}(\lambda)>0$ and $\Delta<0$. As a result, we do not observe stationary with respect to time and heterogeneous with respect to space patterns for the local model (2.5) and (2.6).

We need to check for a range of $k_{x}$ and $k_{y}$ values in $k_{x}-k_{y}$ plane, whether $\operatorname{Re}(\lambda)>0(\lambda$ is a root of the characteristic Eq. (2.15)) for nonlocal model (2.11) and (2.12) and $\Delta<0$ as the nonlocal ranges $\delta_{1}$ and $\delta_{2}$ are increased. For convenience we consider $\delta_{1}=\delta_{2}=\delta$. As we increase $\delta$ from 0.001 to 0.1 to 2 , the $\operatorname{Re}(\lambda)>0$ for two very small regions in the $k_{x}-k_{y}$ plane at $\delta=1.966$. Consequently $\Delta<0$ occurs at those two regions. This means, we have just crossed the Turing bifurcation threshold $\delta_{T}=1.965$ at which $\Delta=0$. We have plotted the surfaces of $\operatorname{Re}(\lambda)$ and $\Delta$ for $\delta=1.9$ and $\delta=2$ in Fig. 2 . Fig. 2 (a) shows that for $\delta=1.9, \operatorname{Re}(\lambda)<0$ and $\Delta>0$ for all the $k_{x}$ and $k_{y}$ values in $k_{x}-k_{y}$ plane. Whereas for $\delta=2$ as shown in Fig. 2(b), $\operatorname{Re}(\lambda)>0$ and $\Delta<0$ in two very small regions in $k_{x}-k_{y}$ plane, the remaining parts still giving $\operatorname{Re}(\lambda)<0$ and $\Delta>0$. We check the plots of surfaces of $\Delta_{k_{x}}$ and $\Delta_{k_{y}}$ which give zero values for major area of the $k_{x}-k_{y}$ plane but we have not kept the figures in the text. When we simulate the nonlocal model (2.11) and (2.12) for $\delta=2$, we get stationary hotspot pattern for both prey and predator populations. As we increase $\delta=3,4,5$, hotspot pattern appears but the number of spots decrease with increasing nonlocal range.

Similarly for $\delta=6$ we obtain the Turing bifurcation threshold to be $d_{2 T}=0.125$. Thus, for the values of $d_{2}>d_{2 T}=0.125$, two-dimensional stationary patterns can be observed for the nonlocal model (2.11) and (2.12). The local spatio-temporal model however is unable to produce any stationary pattern and homogeneous steady state is stable under small amplitude heterogeneous perturbation. For $d_{2}=0.8$, there exist pairs $\left(k_{x}, k_{y}\right)$ for which the real part of the eigenvalue is positive as shown in Fig. 3(a). The wave number for which the real part of the eigenvalue is positive provides the fastest growing mode and lead to formation of the stationary hotspot pattern (see Fig. 3). We explore pattern formation for both the local and nonlocal models in the next two subsections in details. 


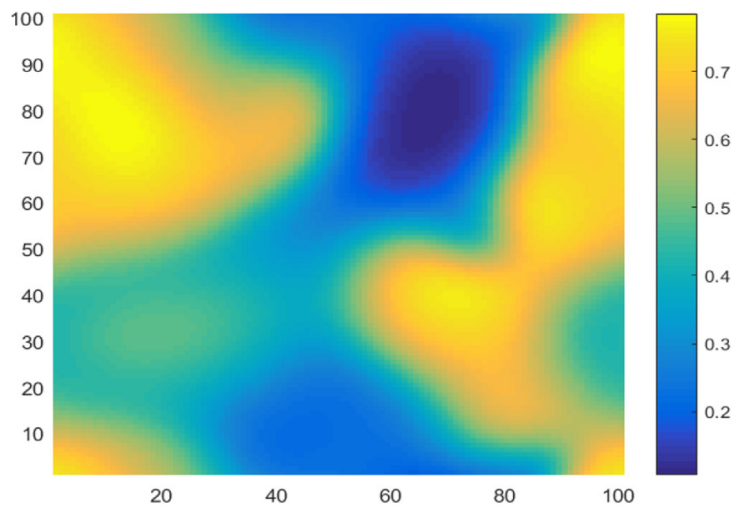

(a)

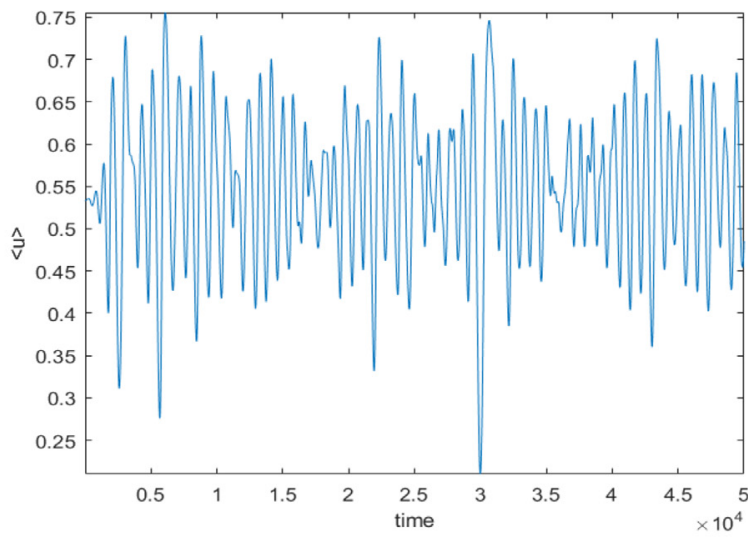

(b)

Fig. 5. Patterns observed for the prey population for $\beta=0.35$ : (a) snapshot of spatio-temporal chaos exhibited by the local model; (b) corresponding spatial average of the prey population density plotted with respect to time.

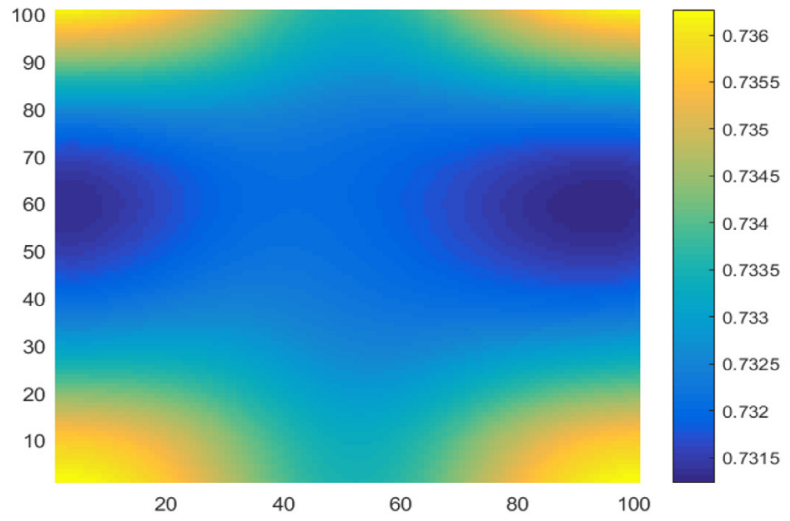

(a)

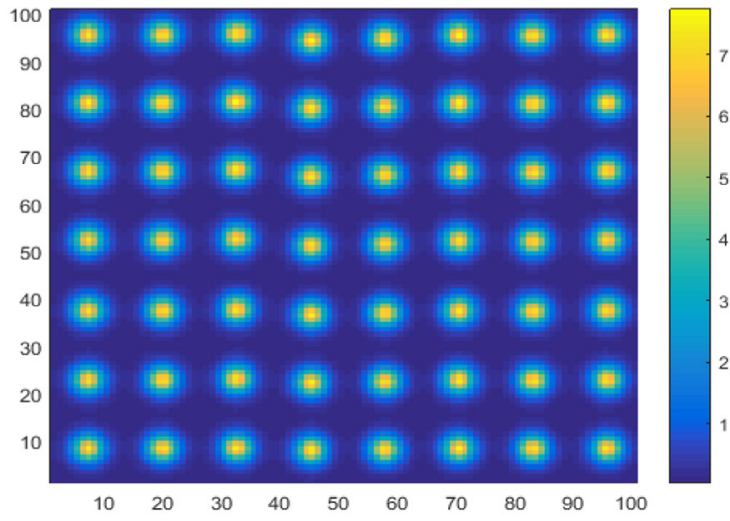

(b)

Fig. 6. Patterns observed for the prey population for $\beta=0.3445$ : (a) snapshot of a periodic solution exhibited by the nonlocal model for $\delta=5$; it is nearly homogeneous in space but oscillatory in time; (b) stationary hot spot pattern for $\delta=6$.

\subsection{Patterns shown by local model (2.5) and (2.6) in two dimensions}

In this subsection we discuss some patterns produced by the local model (2.5) and (2.6). The parameter values are taken to be $a=1, b=1, \sigma_{1}=0.1, \alpha=0.4, \kappa=0.4, \sigma_{2}=0.2 d_{1}=0.1, d_{2}=0.1$. Parameter $\beta$ is changed to study the pattern formation scenario. Stationary patterns are not observed while time depending patterns, mainly periodic and aperiodic, are found for proper parametric choices. For $\beta=0.3445$ we observe a periodic in time solution of the local model (2.5) and (2.6). Its spatial variation is weak. Fig. 4(a) shows a snapshot of the prey distribution, and Fig. 4(b) its spatial average as a function of time. The corresponding predator population shows similar patterns.

As the parameter $\beta$ is increased from $\beta=0.3385$, the system (2.5) and (2.6) generates oscillatory solutions and then spatio-temporal aperiodic solutions for $\beta \geq 0.3475$. Spatio-temporal chaos is presented for the local model for $\beta=0.35$ as shown in Fig. 5(a). In Fig. 5(a) and (b), the snapshot of pattern shown by the prey population along with the time evolution of the spatial average of prey density confirms the behaviour of solutions to be aperiodic. These are the two patterns exhibited by the local model (2.5) and (2.6) in presence of diffusion terms. It is interesting to note that for the values of $\beta$ for which total extinction of both prey and predator population is observed in the temporal model (2.1) and (2.2) (see Fig. 1), reaction-diffusion model (2.5) and (2.6) manifests aperiodic behaviour and imply the coexistence.

\subsection{Effects of nonlocal interaction}

In this subsection we discuss two-dimensional patterns described by the nonlocal model (2.11) and (2.12) and how the patterns generated by the local model (2.5) and (2.6) evolve under the effect of nonlocal interaction. For convenience, we 


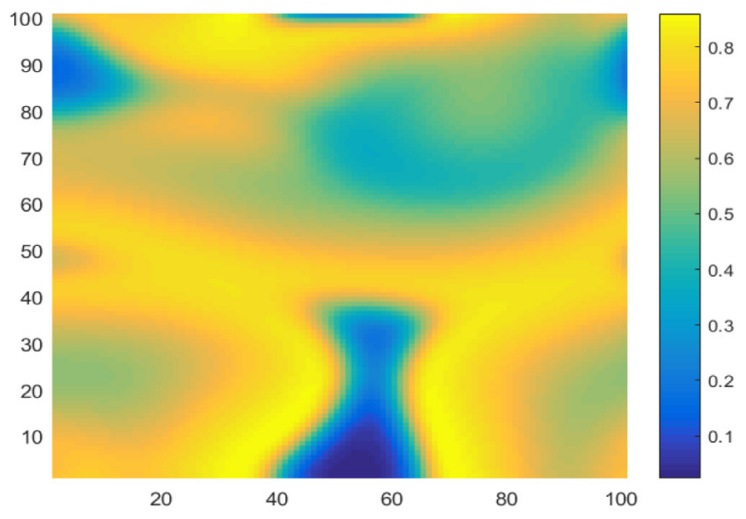

(a)

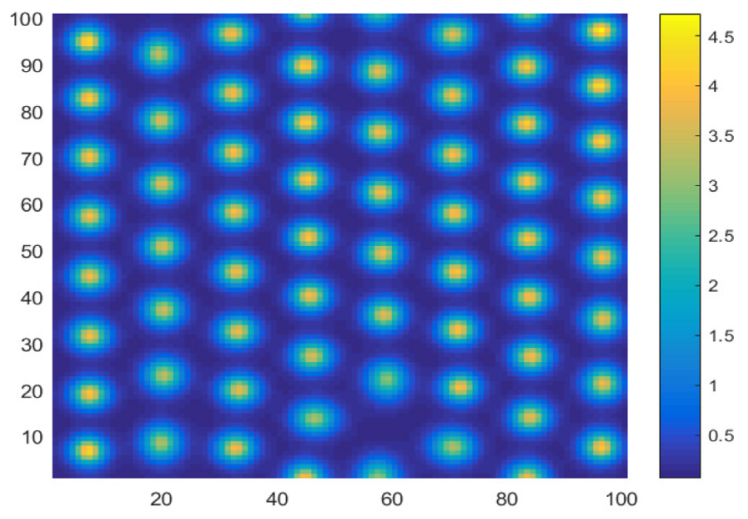

(b)

Fig. 7. Patterns observed for the prey population for $\beta=0.35$ : (a) snapshot of spatio-temporal chaos exhibited by the nonlocal model for $\delta=5$; (b) stationary hot spot pattern for $\delta=6$.

keep $\delta_{1}=\delta_{2}=\delta$. Periodic with respect to time solution observed for the local model (2.5) and (2.6) for $\beta=0.3445$ changes to Turing patterns for $\delta=\delta_{1}=\delta_{2}>6$ (see Fig. 6). As the nonlocal range is increased, the solution retains its homogeneous in space but periodic in time nature till $\delta=\delta_{1}=\delta_{2}=5$ as shown in Fig. 6(a) but as $\delta$ is increased further, the solution subsides into stationary hot spot pattern (see Fig. 6(b)). The corresponding predator population shows similar patterns.

Spatio-temporal chaos observed for the local model for $\beta=0.35$ evolves to Turing patterns for $\delta=\delta_{1}=\delta_{2}>5$ (see Fig. 7). As the nonlocal range is increased, it retains its spatio-temporal aperiodic nature till $\delta=\delta_{1}=\delta_{2}=5$ as shown in Fig. 7(a) but as $\delta$ is increased further, the solution subsides into stationary hot spot pattern (see Fig. 7(b)). The corresponding predator population also shows similar patterns. We have not presented these figures here for the sake of brevity.

\section{Conclusion}

Prey-predator interaction with prey-dependent functional response and constant intrinsic death rate for predators are mostly known as Gauss type models [30]. Interacting prey-predator models within heterogeneous environment and with Gauss type reaction kinetics are unable to produce any stationary heterogeneous distribution of both the species. Such models can support stable coexistence of both the species when they are distributed over space homogeneously. In the presence of space dependent population distribution and Gauss type reaction kinetics, one can find time dependent patterns like travelling wave, wave of invasion, spatio-temporal chaos and some transient patterns [5,21]. Prey-predator model with Rosenzweig-MacArthur reaction kinetics, constant death rate for predator and movement of both the species due to self diffusion only - is unable to produce any Turing pattern. Recently we have shown that incorporation of nonlocal competition in prey population to have access to nearby resources can lead to stationary heterogeneous distribution of prey and predator for a wide range of parameter values [5]. Our investigation was based upon a reaction-diffusion model with nonlocal interaction and one-dimensional space. Here we extend our previous work to a similar type of problem but with two-dimensional space. We have considered a modified growth law for prey population, in the absence of predator, following the approach proposed in Banerjee et al. [1], Petrovskii et al. [20] to justify the two-sex population growth.

The basic model considered in this analysis is relatively new and hence firstly we have obtained the local stability an bifurcation of homogeneous steady-states. Existence of two types of patterns, homogeneous in space but oscillatory in time and spatio-temporal aperiodic pattern exhibited by the reaction-diffusion model is demonstrated in detail. Main contribution of this work is the derivation of Turing instability condition and illustration of resulting stationary pattern through numerical simulation for an integro-differential equation model with same reaction kinetics. Here we have considered two dimensional space and hence the kernel function involved with the nonlocal interaction term is modified accordingly. Small range of nonlocal interaction can not affect the pattern formation scenario significantly, however, reasonable long range of nonlocal interaction leads to spot patterns which are stationary in time. The basic reaction kinetics is valid for prey and their specialist predator, as a result the spatial patterns produced by the reaction-diffusion model support the mechanism that predator follows prey density. In other words, depending upon the strength of interactions, prey can move from one location to other location in an irregular fashion and the specialist predators follow the changing prey density. For integrodifferential model, the movement of prey individuals to nearby food enriched locations for over a short time scale leads to stationary patches of prey. Reasonable amount of food source for the specialist predator also leads to the stationary patches of predators. This mechanism is reflected through the spot patterns produced by prey and their specialist predator. High density of prey and predator at the same spatial locations indicate their coexistence. 
As a first attempt towards the spatio-temporal pattern formation by a prey-predator model with nonlocal interaction among prey and two-dimensional space, we restrict ourselves to the illustration of stationary patterns produced by the prey and predator population. The associated homogeneous model exhibit a limited variety of dynamics either stable or oscillatory coexistence along with the extinction of one or both the species. We have demonstrated that the consideration of nonlocal interaction for favorable resources by the prey can lead to stationary coexistence of both the species. Abundance of favorable resources for prey and reasonable density of prey as main food source for predator can leads to stationary patches of both the species over their habitats. It is important to mention here that the satisfaction of Turing instability condition for the model with two-dimensional nonlocal kernel leads to spot patterns. The admissible range of nonlocal interaction leading to stationary patches is determined by the strength of reaction kinetics and rates of diffusivity of both the species. Our future goal will be to address the pattern formation scenarios for the model which exhibit spot and labyrinthine patterns with nonlocal interaction terms.

\section{Credit author statement}

We hereby declare that the authors have equally contributed to the work.

\section{Declaration of Competing Interest}

Authors declare that they have no conflict of interest.

\section{Acknowledgements}

The first author is supported by Department of Atomic Energy, India. The second author is supported by the Ministry of Science and Higher Education of the Russian Federation: agreement no. 075-03-2020-223/3 (FSSF-2020-0018). We are thankful to Prof. M Banerjee (IIT Kanpur, India) for his valuable suggestions.

\section{References}

[1] Banerjee M, Mukherjee N, Volpert V. Prey-predator model with a nonlocal bistable dynamics of prey. Mathematics 2018;6(41):1-13.

[2] Turing AM. The chemical basis of morphogenesis. Philos Trans R Soc Lond B 1952;237:37-72.

[3] Amarasekare P. Interactions between local dynamics and dispersal: insights from single species models. Theor Popul Biol 1998;53(1):44-59.

[4] Banerjee M, Volpert V. Prey-predator model with a nonlocal consumption of prey. Chaos 2016;26:083120.

[5] Banerjee M, Volpert V. Spatio-temporal pattern formation in Rosenzweig-McArthur model: effect of nonlocal interactions. Ecol Complex 2017;30:2-10.

[6] Baurmann M, Gross T, Feudel U. Instabilities in spatially extended predator-prey systems: spatio-temporal patterns in the neighborhood of Turing-Hopf bifurcations. J Theor Biol 2007;245:220-9.

[7] Cantrell RS, Cosner C. Spatial ecology via reaction-diffusion equations. London: Wiley: 2003.

[8] Courchamp F, Clutton-Brock T, Grenfell B. Inverse density dependence and the Allee effect. Trends Ecol Evol 1999;14(10):405-10.

[9] Dunbar SR. Travelling wave solutions of diffusive Lotka-Volterra equations. J Math Biol 1983;17:11-32.

[10] Fasani S, Rinaldi S. Factors promoting or inhibiting turing instability in spatially extended prey-predator systems. Ecol Model 2011;222:3449-52.

[11] Genieys S, Volpert V, Auger P. Pattern and waves for a model in population dynamics with nonlocal consumption of resources. Math Model Nat Phenom 2006;1(1):63-80.

[12] Gourley SA, Britton NF. A predator-prey reaction-diffusion system with nonlocal effects. J Math Biol 1996;34:297-333.

[13] Klausmeier CA. Regular and irregular patterns in semiarid vegetation. Science 1999;284:1826-8.

[14] Levin SA, Segel LA. Hypothesis for origin of planktonic patchiness. Nature 1976;259:659.

[15] Jankovic M, Petrovskii S. Are time delays always destabilizing? Revisiting the role of time delays and the Allee effect. Theor Ecol 2014;7:335-49.

[16] Murray JD. Mathematical biology II. Heidelberg: Springer-Verlag; 2002.

[17] Medvinsky A, Petrovskii S, Tikhonova I, Malchow H, Li BL. Spatiotemporal complexity of plankton and fish dynamics. SIAM Rev 2002;44:311-70.

[18] Aydogmus O. Patterns and transitions to instability in an intraspecific competition model with nonlocal diffusion and interaction. Math Model Nat Phenom 2015;10(6):17-19.

[19] Okubo A, Levin S. Diffusion and ecological problems: modern perspectives. Berlin: Springer; 2001.

[20] Petrovskii S, Blackshaw R, Li BL. Consequences of the Allee effect and intraspecific competition on population persistence under adverse environmental conditions. Bull Math Biol 2008;70(2):412-37.

[21] Petrovskii SV, Malchow H. A minimal model of pattern formation in a prey-predator system. Math Comput Model 1999;29:49-63.

[22] Segel LA, Jackson JL. Dissipative structure: an explanation and an ecological example. J Theor Biol 1972;37:545-59.

[23] Sen D, Banerjee M, Ghorai S. Allee effect in prey versus hunting cooperation on predator - enhancement of stable coexistence. Int J Bifurc Chaos 2019;29:1950081.

[24] Sherratt JA, Eagan BT, Lewis MA. Oscillations and chaos behind predator-prey invasion: mathematical artifact or ecological reality? Philos Trans R Soc Lond B 1997;352:21-38.

[25] Shigesada N, Kawasaki K. Biological invasions: theory and practice. Oxford: Oxford University Press; 1997.

[26] Volpert V. Branching and aggregation in self-reproducing systems. ESAIM 2014;47:116-29.

[27] Volpert V. Pulses and waves for a bistable nonlocal reaction-diffusion equation. Appl Math Lett 2015;44:21-5.

[28] Volpert V. Elliptic partial differential equations. Reaction-diffusion equations, 2. Birkhauser; 2014.

[29] Volpert V, Petrovskii SV. Reaction-diffusion waves in biology. Phys Life Rev 2009;6:267-310.

[30] Freedman IH. Deterministic mathematical models in population ecology. Pure and Applied Mathematics; 1982. 